\title{
OpenGL 3D crowd evacuation simulation at universiti tun hussein onn malaysia (UTHM) hostel
}

\author{
Muhammad Naqib bin Jamaludin ${ }^{1}$, Shamsul Mohamad ${ }^{2}$, Mohd Shahrizal Sunar ${ }^{3}$, Khalid Isa ${ }^{4}$ \\ Rafizah Mohd Hanifa ${ }^{5}$, Fawwaz Mohd Nasir ${ }^{6}$, Shaharil Mohd. Shah ${ }^{7}$ \\ ${ }^{1,2,4,7}$ Faculty of Electrical and Electronic Engineering, Universiti Tun Hussein Onn Malaysia, Malaysia \\ ${ }^{2,3}$ UTM-IRDA Digital Media Centre, Media and Game Innovation Centre of Excellence, \\ Universiti Teknologi Malaysia, Malaysia \\ ${ }^{2,3}$ School of Computing, Faculty of Engineering, Universiti Teknologi Malaysia, Malaysia \\ ${ }^{5,6}$ Centre for Diploma Studies, Universiti Tun Hussein Onn Malaysia, Malaysia
}

\section{Article Info \\ Article history:}

Received Jan 20, 2019

Revised Mar 23, 2019

Accepted Apr 9, 2019

\section{Keywords:}

3D Crowd

Crowd evacuation

OpenGL

Social force model

\begin{abstract}
Crowd simulation is the process of simulating characterized agents or entities using computer application to analyse it in virtual scene or virtual environment. This paper investigates the best route path for agents to act in avoiding the fire hazards with different designated type of stairs in shop lots that were converted to hostel dormitory for students. 3D social force agent's model and 3D fire hazards were designed in Microsoft Visual Studio C++ software and OpenGL library. A research was conducted using social force model behaviour and were taken by 10 and 15 agents to analyse the time taken to complete the evacuation process. The acceleration produced where it is related with route path taken by agents, interaction forces of agents and interaction forces of wall are the main research system to analyse agents' behaviour during simulation. Different simulations have been used to determine the best and fastest route taken by agents. In summary, the lower the number of agents, the lower the time allocated by agents to complete the evacuation. Finally, less number of agents using the designated straight stairs gave a lower time to complete evacuation process and reached high level of security to avoid being exposed to fire hazards.
\end{abstract}

Copyright (c) 2019 Institute of Advanced Engineering and Science. All rights reserved.

\section{Corresponding Author:}

Shamsul Mohamad,

Faculty of Electrical and Electronic Engineering,

Universiti Tun Hussein Onn Malaysia,

86400 Parit Raja, Batu Pahat, Johor Darul Ta'zim, Malaysia.

Email: shamsulm@uthm.edu.my

\section{INTRODUCTION}

Crowd simulation is the process of simulating some of the characterized agents in interacting with the environment and surroundings. It is also known as the dynamic movement involving a large number of character and entities. Besides, it is commonly used to create virtual scenes for visual media such as gaming interface, crisis training, architecture, urban planning and evacuation simulation [1]. The introduction of this crowd also have its own desired motion and behavioural of crowds of people. Furthermore, crowd simulations have some techniques in order to know the type of characters being present in the social force model [2-3].

New parameters such as the social force can be related with crowd analysis to interpret the data gained about the moving objects. The social force model describes the motion of pedestrians in a crowded area resulting from the combination of the desire to reach their destination via the shortest path and some interaction forces. This paper aims to realize the simulation of crowd behaviour in three-dimensional (3D) making it more immersive to study and observe the crowds' behaviour. The 3D crowd simulation is one of the new crowd simulation technology, introduced in recent years, to give a clearer appearance of agents' behaviour. This paper focuses on simulating the crowd movement at the dormitory above the shop lots, which is a common feature in all universities and other higher learning institutions. By focusing on this 
particular aspect, institution administrators could ideally design, evaluate, and improve dormitory layouts and safety features to ensure it meets the minimum safety requirements for emergency egress.

These shop lots are owned and leased by the universities to fulfil students' need for groceries, stationaries and other items. The structural design of the building and safety features that exist in a shop lot area can affect the route path people or agents will take during an evacuation. The best route for agents to complete the evacuation process depends on the type of staircase in the shop lot. The type of hazards used varies from a simple hazard such as a spreading fire to a more complicated hazard such as smoke propagation and added level of toxicity[4].

There is an availability of working simulation through the real system with real world with 3Dlooking image. The best feature is to create a model and simulation related to the hazard situation [5]. Visual view shows a clearer picture of the beginning when comparing with a practical view which can reduce development time and cost. Through the simulation, students which are acting as agents can be tested in realistic scenarios such as in danger and more strategic flow process. The simulation is to describe human behaviour in real hazard situations as well as the details of the crowd behaviour modelling method. This situation can be done by doing an early preparation by using crowd simulation to avoid tragedies or untoward incidents from happening in the dormitory. Therefore, this paper presents a 3D crowd simulation evacuation at the hostel above shop lots using the Open Graphics Library (OpenGL).

\section{LITERATUREREVIEW}

Modelling and simulating crowd evacuation under emergency condition is important as it could be used as one of the tool to analyse and assess safety precautions. Currently, there are many simulation models in research and development that deal with crowd evacuation.

Pedestrians have been empirically studied for more than four decades [6-8]. Since the 1990s, there have been a fast growing interest in understanding and modelling pedestrian behaviour. One important aspect of this is related to crowd management, especially during emergency situations, whereby pedestrians have to be routed and evacuated through the building in a fast and efficient manner [9-10]. Classical, qualitative manners of flow analysis are no longer sufficient. Therefore, simulation models are needed to optimize the proposed infrastructural design and to test and improve crowd management inside an existing or planned infrastructure. Pedestrian simulation is a new paradigm to evaluate the outcomes of various designs, controls and policies which can be done through mathematical equations or computer programs. It is easy to change the layout and rules of facilities within the simulation. Unfortunately, in reality, it is very costly to make such a change. Therefore, simulation has been a great tool to experiment many behavioural rules and in creating various what-if scenarios.

Many pedestrian models have been developed in the past decades. Figure 1 shows the organisation of pedestrian simulation model. Basically, there are two main approaches: continuous and discrete. Continuous approach is used for a long term and steady state effect of the system while the discrete approach generates dynamic entities as in real life such as the pedestrians. Discrete approach can be further distinguished into two levels: level of interaction and level of assemblage. The former level can be further divided into macroscopic, mesoscopic and microscopic while the latter level consists of individual (multi-agent) and group of individuals.

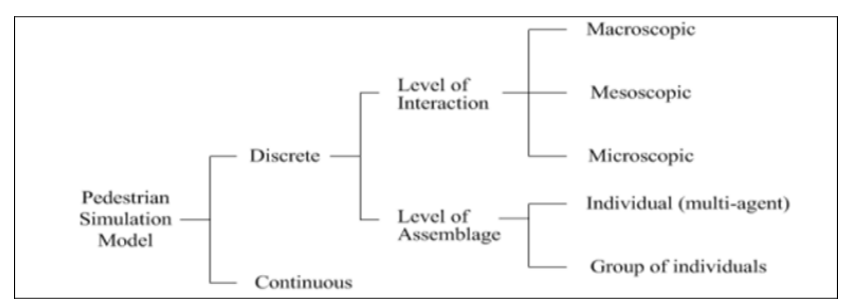

Figure 1. Category of pedestrian simulation model

Microscopic model is preferable by most of the modeller due to its ability in detailing the interaction between the pedestrians themselves and between pedestrians and the environment. This is accomplished through a set of parameters and models that produce agents' intention to move in a certain velocity or acceleration [11]. Table 1 presents a summary of the differences between three models under interaction level. 
Table 1. Summary of Pedestrian Model Approach

\begin{tabular}{ccl}
\hline $\begin{array}{c}\text { Pedestrian Flow } \\
\text { Models }\end{array}$ & & \multicolumn{1}{c}{ Descriptions } \\
\hline $\begin{array}{c}\text { Macroscopic } \\
\text { models[12] }\end{array}$ & - & $\begin{array}{l}\text { Do not make distinctions between individual nor describe their individual behavior but consider the flow } \\
\text { in terms of density, average velocity and flow patterns. }\end{array}$ \\
$\begin{array}{c}\text { Mesoscopic models } \\
\text { Do not consider each pedestrian individually but instead consider the overriding characteristics (e.g. } \\
\text { velocity distribution) }\end{array}$ \\
models[13] & - & Consider individual pedestrian behaviour separately. \\
Behaviour of concern is on the interactions with other pedestrians in the crowd.
\end{tabular}

Helbing and Molnar proposed a method which is called social force. The Social Force Model (SFM) is a physical model based on the social field theory. According to them, human behaviours are complicated and can be viewed as almost chaotic by numerous parties especially during emergency. The idea behind social force is to find a set of basic rules based upon behavioural aspects.

SFM has been successful in reproducing several collective pedestrian flow phenomena and in analysing different characteristics of pedestrian flow. The importance of this model is that it deals specifically with panic situations and several simulations of case studies on panic escape from a room have been performed [14]. Unlike MFM which have abstract/vague values, the parameters used in the SFM model have physical meaning and can be measured. The model is also flexible for simulating both normal behaviour and panic behaviour of pedestrians.

Similarly as in the previous models, SFM has not been validated using real world data for panic situations. Besides, the variations in microscopic pedestrian characteristics have not been considered and thus, making it insufficient to simulate complex scenarios when route finding is important. In reality, pedestrians may formulate better escape strategies such as moving between two other pedestrians to find a quieter route which have not been taken into account by this social force models.

Agent-based models began as a set of ideas and techniques that were used to implement models of complex adaptive systems [15]. The basic definition of agent-based is a modelling approach that models a system as a set of individual, autonomous and interacting agents. There is no precise definition of an agent and some researcher consider any types of independent component to be an agent, while others suggest that a component must be adaptive to be defined as an agent. In general, agents are considered to have four essential properties: self-contained, autonomous, interacts with other agents and has a state that can vary over time.

Table 2 shows the comparison of crowd modelling technique in terms of individuals/group, level of interactions, space and time and also computationally cost. In term of individuals/group, some techniques considered pedestrians as homogeneous individuals whereas other techniques viewed pedestrians as heterogeneous individuals (groups) based on different characteristics (e.g., gender, age, psychology). In term of level of interactions, in some models, pedestrian dynamics is modelled on a microscopic scale where collective phenomena emerge from the complex interactions between many individuals (self-organizing effects). Whereas, in other models, the pedestrian dynamics is modelled on a macroscopic scale when a crowd of pedestrians is considered as a whole [16-18].

Table 2. Crowd Modelling Techniques

\begin{tabular}{ccccc}
\hline Technique & Individuals / Group & Level of Interactions & Space \& Time & Computationally Cost \\
\hline Cellular Automata [19] & Homogenous & Microscopic & Discrete & Low \\
Lattice Gas [20] & Homogenous & Microscopic & Discrete & Low \\
Social Force [21 - 23] & Homogenous & Microscopic & Continuous & Low \\
Fluid Dynamics [24] & Homogenous & Macroscopic & Continuous & Low \\
Agent-based [25 - 26] & Heterogenous & Microscopic & Discrete / Continuous & High \\
Game Theory [27] & Homogenous & Microscopic & Discrete & High \\
\hline
\end{tabular}

\section{METHODOLOGY}

3.1. Design a Dormitory Layout

The layout of the infrastructure is important in order to analyse and understand the behaviour of an agent going through every obstacle. Figure 1 shows the layout which includes common facilities such as toilets, laundry and drying area, rooms, bed, desks, closets and living room. The layouts are created using OpenGL's GL_LINES geometric primitive by specifying a set of coordinates. These specific coordinates determine the length of a wall which is plotted on the $\mathrm{x}$ and $\mathrm{y}$-axes. The coordinates also determine which appropriate location to place the facilities that were used. Based on this layout, it falls under a wall.cpp configuration and declaration. There are specific coordinate ranges that were set up from the start to the end 
of wall layout which give scale vectors. For simplicity and computational speed, the scale vector in the simulation relates to social force model acts by agents to the desired safe area. The setPoint function which is used to define a wall layout takes four parameters: $x_{1}, y_{1}, x_{2}, y_{2}$.

In this section, it is explained the results of research and at the same time is given the comprehensive discussion. Results can be presented in figures, graphs, tables and others that make the reader understand easily [11-15]. The discussion can be made in several sub-chapters.

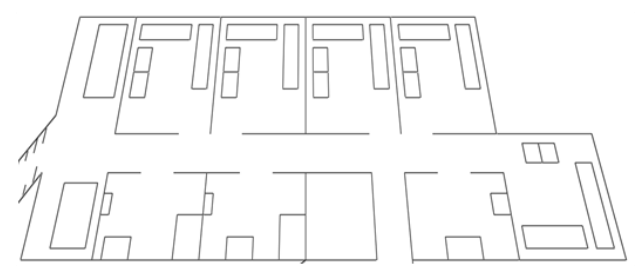

Figure 2. Design of dormitory layout

The relative vector for wall starts and ends specified ideal layout without the complexity and increasing the time taken even though there are too many of agents were created.

\subsection{Agent Shape and Motion}

Agents in this paper are represented using a simple cylinder. Since OpenGL does not have a primitive function to render a cylinder, this paper uses a combination of isosceles triangles (using GL_TRIANGLES) to form two separate circles, which are joined by a set of rectangles (using GL_QUADS) to form the cylinder's shaft. The angle between the two sides of the triangle which are equal in length are carefully determined to balance between rendering performance and jaggedness. A function to render this set of triangles and rectangles is created, receiving these basic parameters: the coordinates $x$ and $y$, the radius and the height.

There are many crowd simulation formulations that can be implemented to represent the behaviour of agents such as agent-based model, game-theoretic model, fluid-dynamics model, cellular automata models, lattice gas models and social force model. In this work, social force model uses as modelling method because it is able to reproduce many emergent phenomena observed in real crowds. Basically, the social force model can be represented in (1).

$$
\text { acceleration }=\text { driving Force }+ \text { agent Interact Force }+ \text { wall Interact Force }
$$

The acceleration qualify towards the desired velocity of motion and how agents interacting with other agents without any collisions with each other. The force between agents and walls will appear if the exact point were set up. A sensory stimulus causes a behavioral reaction that depends on personal aim which related to the set of behavioral alternatives with the objective of utility maximization. The movement of agents is dependent on a driving force (driving Force) and is affected by two interaction forces (agent Interact Force and wall Interact Force) where it assigns a specific path to each agent in determining the direction of their movement and the resulting interaction force during the simulation.

In the beginning of the movement generated by the agent, the agents will keep a certain distance from the border with wall structures. Thus, a smooth movement and flow process produced by the agents will allow fixed acceleration. Figure 3 and 4 show the flow of agents before and after the simulations.

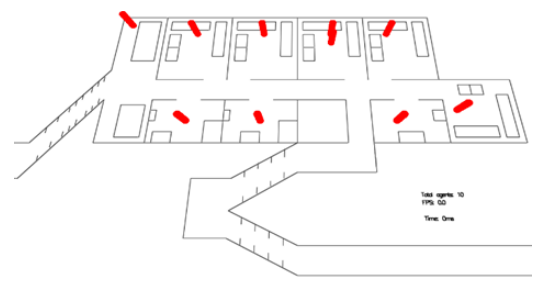

Figure 3. Initial state before agents moving to the set path

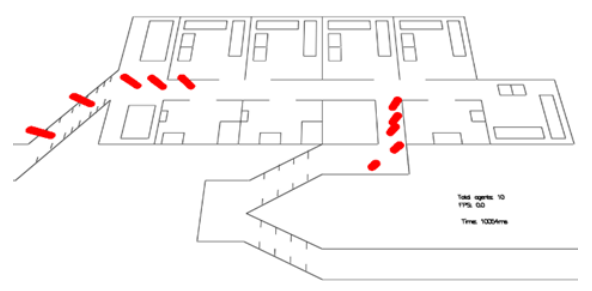

Figure 4. After agents moving to the path that has been set up 


\subsection{D Hazards as a Barrier}

Hazards are important components to observe crowd behaviour in determining their initial set path to the safe place to avoid any unwanted incidents. Agents will concern with the hazards activities and look for solutions to stay away from it. Based on the conception of social force model, the parameter between agent and hazards can be further separated by creating a random accident in the dormitory. Besides, this event will allow an observation to be made either it is affected by the agents' motion or psychological principle.

Relating hazards with social force, there are another newly created agents' forces that can be seen because it might delay agents' flow process, affect social force acceleration and the desired velocity and motion. Some observations can also be made as a result of putting the $3 \mathrm{D}$ fire hazards as a barrier, which can create an agent's response

\section{RESULTS AND ANALYSIS}

Based on the various simulations, there are three different situations of fire hazards which are at the first entrance door, at the corridor and at the back door. In the simulation, the location of fire hazards has been set in the dormitory by using OpenGL library as shown in Figure 5.
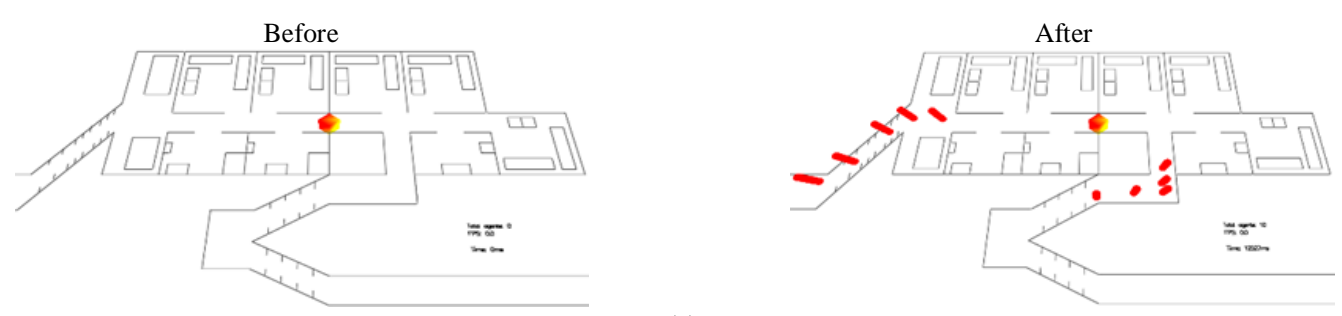

(a)
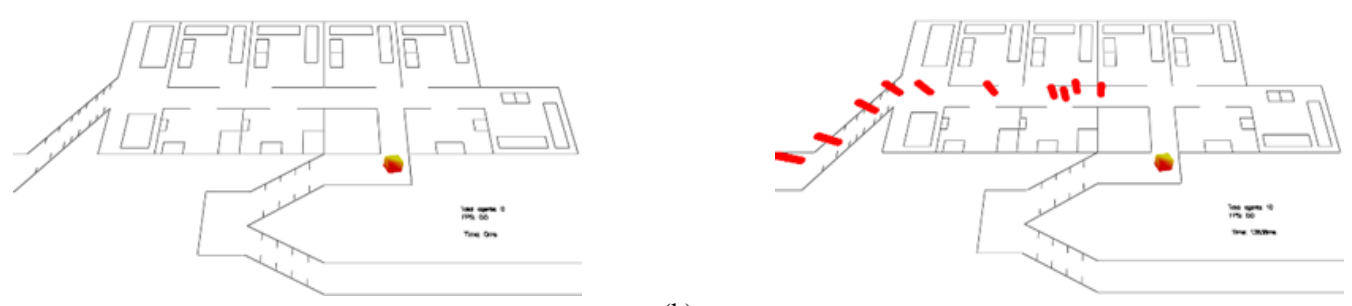

(b)
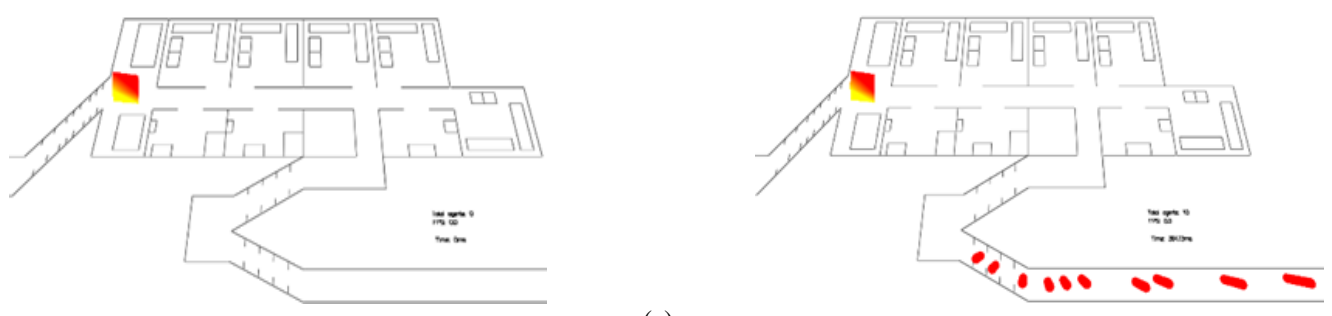

(c)

Figure 5. Agent position before and after evacuation process

In realizing the simulation process, there are 10 agents involved in the evacuation process. As a comparison of crowd analysis, 15 agents were also simulated to find the differences between the time and agents' behavioural. These 10 and 15 agents have different coordinate locations and it will determine the simulation of movements for them to move to a safer place. The agents will act in determining its designated directions to avoid any bad incidents.

There are several simulated time duration to investigate the appropriate average time for the agents to move from the initial coordinate to the evacuation point. From the observation, Table 3 recorded the average time taken to complete a simulation on different locations of fire hazards and the number of agents involved in each type of stairs. 
Table 3. Average Time Recorded for Three Simulations on Different Locations of Hazards and Different Types of Stairs

\begin{tabular}{clcc}
\hline Number of Agents & Location of Fire Hazards & Type of Stairs & Average Time (s) \\
\hline 10 & Back Door Area & U-shaped Stairs & 40.142 \\
10 & Front Door Area & Straight Stairs & 36.894 \\
10 & Corridor & U-shaped Stairs \& Straight Stairs & 34.831 \\
15 & Back Door Area & U-shaped Stairs & 41.684 \\
15 & Front Door Area & Straight Stairs & 38.472 \\
15 & Corridor & U-shaped Stairs \& Straight Stairs & 35.071 \\
\hline
\end{tabular}

From the table, it is shown that the average time for hazards located at the back door area is 40.142s. Furthermore, the best time required for 10 agents to go through the straight stairs at the back door while hazards are located at the front door is 36.894s. The time recorded when hazards occur in corridor for 10 agents are lower than fire hazards occur at the back door and main door area. This is due to the number of agents that is divided into two where 5 agents go through the back door using straight stairs and 5 others agents go through the front door using U-shaped stairs. Therefore, the congestion between agents decreases.

Besides, the simulated time recorded difference results if the dormitory becomes crowded. Congestion between agents to complete has resulted in the evacuation process to take longer time. In addition, when the fire hazards occur at the back door, the agent needs $41.684 \mathrm{~s}$ if using the U-shaped stairs to complete the evacuation process. On the other hand, if the fire hazards are located at the front door, the average time recorded is $38.472 \mathrm{~s}$ for 15 agents to complete the evacuation process if using the straight stairs. The time taken is faster than the time taken when the fire hazards occur at the back door area. It is because the back door is an emergency door and also a safety path where the stairs are different from the stairs that were designed for the main door. The average time for 15 agents when the fire hazards are located at the corridor is $35.071 \mathrm{~s}$ in which the time difference between the 10 and 15 agents is only $0.24 \mathrm{~s}$. The distribution of agents lead to a smooth completion during the evacuation process.

\section{CONCLUSION}

In conclusion, this work has developed 3D virtual crowd models to prove that the simulation of crowd behaviour can be realized in computer vision techniques in analysing the crowd behaviour. In order to achieve the goals, the simulations were done in three different fire hazards simulations which were located at random coordinates. Furthermore, to verify the simulation results, the animation of agents' movement in the crowd to avoid fire hazards were captured. From the three different simulations, it is observed that the locations of fire hazards affect the average time and the path direction taken by the agents to reach the safer place. Meanwhile, the simulation time for agents to complete the evacuation process differ from the types of stairs to escape the fire hazards where straight stairs recorded faster time than the U-shaped stairs.

\section{ACKNOWLEDGEMENTS}

The authors would like to acknowledge Universiti Tun Hussein Onn Malaysia (UTHM) for the funding of this work under the Tier-1 Research Grant (U849).

\section{REFERENCES}

[1] Liu, Qian. "A Social Force Model for the Crowd Evacuation In A Terrorist Attack." Physica A: Statistical Mechanics and its Applications, Elsevier, vol. 502(C), pages 315-330, 2018.

[2] Mohamad, S., Nasir, F.M., Sunar, M.S., Isa, K., Hanifa, R.M., Shah, S.M., Ribuan, M.N., Ahmad, A., "Intelligent Agent Simulator in Massive Crowd". Indonesian Journal of Electrical Engineering and Computer Science (IJEECS) Vol. 11, No. 2, August 2018, pp. 577 584.

[3] Wang, Peng. "Understanding Social-Force Model in Psychological Principles of Collective Behavior". 2016.

[4] Nasir, F.M. and Sunar, M.S. "A Survey on Simulating Real-time Crowd Simulation". 2015 4th International Conference on Interactive Digital Media (ICIDM), pp.1-5, 2015.

[5] Bellomo, N., Piccoli, B. and Tosin, A. "Modeling Crowd Dynamics from a Complex System Viewpoint", Mathematical Models and Methods in Applied Sciences, 22(2), p. 1230004, 2012.

[6] Helbing, D. and Molnár, P. "Social Force Model for Pedestrian Dynamics”, Physical Review E, 51(5), pp. 428242861995 .

[7] Helbing, D., Farkas, I. and Vicsek, T., "Simulating Dynamical Features of Escape Panic". Nature, 407(6803), pp.487-90. 2000.

[8] Helbing, D. and Johanson, A., "Pedestrian, Crowd and Evacuation Dynamics", pp.6476-6495. 2010. 
[9] Yasir Salih, Mohammed Simsim. "Visual Surveillance for Hajj and Umrah: A Review". IAES International Journal of Artificial Intelligence (IJ-AI) Vol. 3, No. 2, June 2014, pp. 90 104.

[10] Guo, R. et al. "Pedestrians' Behavior in Emergency Evacuation : Modeling and simulation." Chinese Phys. B 2016.

[11] Teknomo, K., "Microscopic Pedestrian Flow Characteristics : Development of an Image Processing Data Collection and Simulation Model", 2002.

[12] Nakhmani, A., Surana, A. and Tannenbaum, A. "Macroscopic Analysis of Crowd Motion in Video Sequences". 53rd IEEE Conference on Decision and Control, pp.1822-1827, 2014.

[13] Nicolas, A., Bouzat, S. \& Kuperman, M. N. "Pedestrian Flows through a Narrow Doorway: Effect of Individual Behaviours on The Global Flow and Microscopic Dynamics". Transportation Research Part B: Methodological 99, 30-43 (2017).

[14] Macal, C.M. and North, M.J., "Teaching Agent-Based Modeling and Simulation”. Simulation Conference. 2010.

[15] Ali Z, Shahzad SK, and Shahzad W. "Performance analysis of support vector machine based classifiers". International Journal of Advanced and Applied Sciences, 5(9): 33-38, 2018.

[16] Azizul Fata, A. Z. et al. "Social Force Model for Pedestrian Movement", Journal of Theoretical and Applied Information Technology, 92(1), pp. 181-189, 2016.

[17] Bellomo, N., Piccoli, B. and Tosin, A. "Modeling Crowd Dynamics from a Complex System Viewpoint", Mathematical Models and Methods in Applied Sciences, 22(2), p. 1230004, 2012.

[18] Duives, D. C., Daamen, W. and Hoogendoorn, S. P. "State-of-the-Art Crowd Motion Simulation Models", Transportation Research Part C: Emerging Technologies. Elsevier Ltd, 37, pp. 193-209, 2014.

[19] Khashoggi, A. et al. "Field Cellular Automata Model". 2018 1st International Conference on Computer Applications \& Information Security (ICCAIS), pp.1-6, 2018.

[20] Bu, F. "Lattice Gas Automata-Based Simulation of Evacuation". 2010 IEEE International Conference on Emergency Management and Management Sciences, (60275018), pp.281-284, 2010.

[21] Ismail, F.S. et al., "Simulation of Crowd Evacuation in Laboratory using Social Force Model". 2018 International Symposium on Agent, Multi-Agent Systems and Robotics (ISAMSR), pp.1-6. 2018.

[22] Mohamad, S. et al. "Making Decision For The Next Step In Dense Crowd Simulation Using Support Vector Machines". Proceedings - VRCAI 2016: 15th ACM SIGGRAPH Conference on Virtual-Reality Continuum and Its Applications in Industry. 2016.

[23] Mohamad S., Nasir F.M., Sunar M.S, Isa K., Hanifa R.M., Shah S.M, Ribuan M.N., Ahmad A., "Intelligent Agent Simulator in Massive Crowd". Indonesian Journal of Electrical Engineering and Computer Science Vol. 11, No. 2, August 2018, pp. 577 58.

[24] Al-nasur, S.J., 2006. New Models for Crowd Dynamics and Control. Available at: http://scholar.lib.vt.edu/theses/available/etd-12152006-143454/.(Thesis)

[25] Huang, L., Gong, J., Li, W., Xu, T., Shen, S., Liang, J., Feng, Q., Zhang, D., \& Sun, J. "Social Force Model-Based Group Behavior Simulation in Virtual Geographic Environments". ISPRS Int. J. Geo-Information, 7, 79, 2018.

[26] Kim, S., Guy, S. J. and Manocha, D. "Velocity-Based Modeling of Physical Interactions in Multi-Agent Simulations", in Proceedings of the 12th ACM SIGGRAPH / Eurographics Symposium on Computer Animation (SCA '13). New York: ACM Press, pp. 125-133, 2013.

[27] Sun, Q. "A Generic Approach to Modelling Individual Behaviours in Crowd Simulation". Ph.D. Thesis. University of Salford, 2013.

\section{BIOGRAPHIES OF AUTHORS}

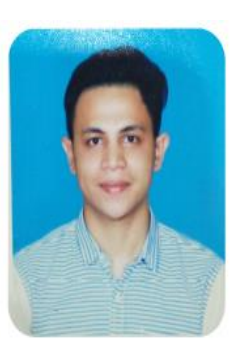

Muhammad Naqib bin Jamaludin obtained his B Eng in Electronic Engineering from Universiti Tun Hussein Onn Malaysia in 2018. Currently, he is Presales Cum Systems Engineer major in Computer \& Networking which proficient in security ELV devices including motion detection, face recognition intelligence and vehicle detection.

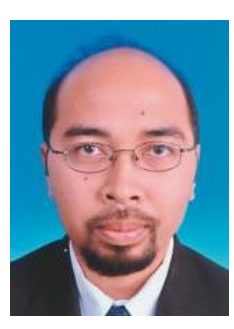

Shamsul Mohamad obtained his BSc in Computer Science from Universiti Teknologi Malaysia in 1999 and MSc in Computer Science from Universiti Sains Malaysia in 2004. Currently, he is Senior Lecturer in the Computer Engineering Department, Faculty of Electrical and Electronic Engineering, Universiti Tun Hussein Onn Malaysia (UTHM). His research interests include crowd simulation, artificial intelligence and Internet of Things. 


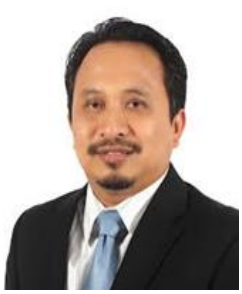

Profesor Dr Mohd Shahrizal Sunar is the Director of Institute of Human Centered Engineering (iHumEn) and Founding Director of Media and Game Innovation Centre of Excellence (MaGICX), Universiti Teknologi Malaysia. He obtained his PhD from National University of Malaysia in 2008. His major field of study is real-time and interactive computer graphics and virtual environment. He served as academic member at Computer Graphics and Multimedia Department, Faculty of Computer Science and Information System, Universiti Teknologi Malaysia since 1999. Since 2009, he had been given responsibility to become the Head of Department and leading the Virtual, Visualisation and Vision research group (UTM ViCubeLab). Dr. Shahrizal is an active professional member of ACM SIGGRAPH, IEEE Computer Society and International Association of Engineers (IAENG). The current research program that he lead are Augmented Reality, Virtual Reality, Gamification, User Interaction and Creative Content Technology. He had published numerous articles in high-impact refereed journals, conference proceedings and technical papers including article in magazines.

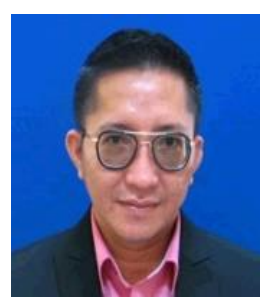

Khalid Isa graduated from the Universiti Teknologi Malaysia, in 2001 with a BSc in Computer Science. He then pursued his MSc. in Computer System Engineering at the Universiti Putra Malaysia, graduating in 2005. Following from this, in 2014, he completed his $\mathrm{PhD}$ degree in Electrical and Electronic Engineering at Universiti Sains Malaysia, with specialization in Computational Intelligence and Underwater Robotics. He is currently a Senior Lecturer in the Computer Engineering Department, Faculty of Electrical and Electronic Engineering, Universiti Tun Hussein Onn Malaysia (UTHM). He is also Head of Career Department in the Student Development Centre, UTHM and Chairman of IEEE Oceanic Engineering Society (OES) Malaysia Chapter. His research interests are underwater robotics, computational intelligence, bio-inspired engineering, internet of things, image processing, control, and autonomous system.

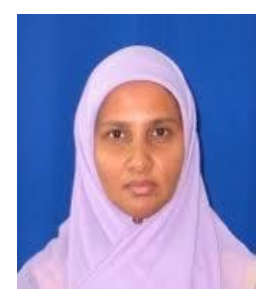

Rafizah Mohd Hanifa obtained her bachelor degree in Computer Science from Universiti Sains Malaysia (USM) in 1999, followed by master degree of Information Technology, Faculty of Information Technology, Universiti Utara Malaysia (UUM), Sintok, Malaysia in 2001. Currently, she is a lecturer at Center for Diploma Studies, Universiti Tun Hussein Onn Malaysia (UTHM), Johor. Her research interests include artificial intelligent, information system and augmented reality.

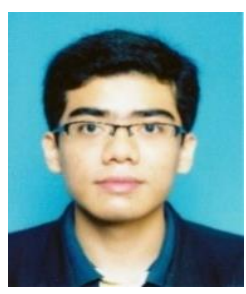

Fawwaz Mohd Nasir obtained his bachelor's degree in Computer Science in 2014, followed by a master's degree in 2017, both from Universiti Teknologi Malaysia (UTM). Currently he is a lecturer at the Centre for Diploma Studies, Universiti Tun Hussein Onn Malaysia (UTHM). His research interests include crowd simulation, collision detection and artificial intelligence.

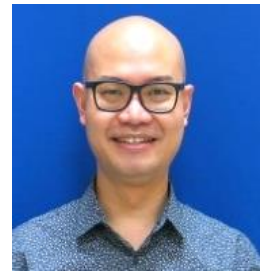

Shaharil Mohd. Shah obtained his B. Eng in Microwave and Communication from Multimedia University (MMU) in 2002. He received his M.Sc in Microwave Engineering and Wireless Subsystems Design from the University of Surrey, UK in 2004 and pursuing his Ph.D in Communication Engineering from the University of Birmingham, UK before graduating in 2016. $\mathrm{He}$ is currently a senior lecturer in the Department of Communication Engineering, Faculty of Electrical and Electronic Engineering, Universiti Tun Hussein Onn Malaysia (UTHM). His area of research includes, but not limited to, design of microwave devices, active antennas measurement and nonlinear characterisation of active devices. 